\title{
A Numerical Study of Combined Use of Two Biocontrol Agents with Different Biocontrol Mechanisms in Controlling Foliar Pathogens
}

\author{
X.-M. Xu, P. Jeffries, M. Pautasso, and M. J. Jeger
}

First author: State Key Laboratory of Crop Stress Biology for Arid Areas, College of Plant Protection, Northwest A\&F University, Yangling, Shaanxi, 712100, P.R. China, and Plant Pathology, East Malling Research, New Road, East Malling, ME19 6BJ, UK; second author: School of Biosciences, University of Kent, Canterbury CT2 7NJ, UK; and third and fourth authors: Division of Biology, Imperial College London, Silwood Park Campus, Ascot, SL5 7PY, UK. Accepted for publication 23 February 2011.

\section{ABSTRACT}

Xu, X.-M., Jeffries, P., Pautasso, M., and Jeger, M. J. 2011. A numerical study of combined use of two biocontrol agents with different biocontrol mechanisms in controlling foliar pathogens. Phytopathology 101:10321044 .

Effective use of biocontrol agents is an important component of sustainable agriculture. A previous numerical study of a generic model showed that biocontrol efficacy was greatest for a single biocontrol agent (BCA) combining competition with mycoparasitism or antibiosis. This study uses the same mathematical model to investigate whether the biocontrol efficacy of combined use of two BCAs with different biocontrol mechanisms is greater than that of a single BCA with either or both of the two mechanisms, assuming that two BCAs occupy the same host tissue as the pathogen. Within the parameter values considered, a BCA with two biocontrol mechanisms always outperformed the combined use of two BCAs with a single but different biocontrol mechanism. Similarly, combined use of two BCAs with a single but different biocontrol mechanism is shown to be far less effective than that of a

Sustainable biological control of plant pathogens depends on effective exploitation of naturally occurring microorganisms. The principal mechanisms involved include mycoparasitism, antibiosis, competition, and induced resistance (IR) $(1,9,36)$; additional mechanisms are hypovirulence mediated through fungal viruses (41) and inhibition of enzymes involved in plant pathogenicity (23). Individual biocontrol mechanisms have been shown to be predominant for some biocontrol agents (BCAs) but there are also many instances where more than one mechanism may operate in a given BCA isolate $(8,47)$. There has been a limited amount of biocontrol success in field crops, most success being achieved in greenhouse cultivation $(13,35)$.

Mathematical modeling can be used to study disease dynamics in relation to BCA dynamics through explicit or implicit consideration of the biocontrol mechanisms $(5,21,25,26)$. Qualitative analysis of a generic model with explicit incorporation of biocontrol mechanisms showed that the rates of a BCA colonizing diseased or healthy plant tissues and the duration of BCA activity are the most important factors in determining the final outcome of a biocontrol system (21).

A range of biocontrol mechanisms may operate in mixed BCA populations $(10,17,18,43)$, although the relative significance of each mechanism is unknown but expected to vary with circumstances. In mixed BCA populations, a further complication is that

Corresponding author: X.-M. Xu; E-mail address: xiangming.xu@emr.ac.uk

doi:10.1094/PHYTO-10-10-0267

(C) 2011 The American Phytopathological Society single BCA with both mechanisms. Disease suppression from combined use of two BCAs was very similar to that achieved by the more efficacious one. As expected, a higher BCA introduction rate led to increased disease suppression. Incorporation of interactions between two BCAs did not greatly affect the disease dynamics except when a mycoparasitic and, to a lesser extent, an antibiotic-producing BCA was involved. Increasing the competitiveness of a mycoparasitic BCA over a BCA whose biocontrol mechanism is either competition or antibiosis may lead to improved biocontrol initially and reduced fluctuations in disease dynamics. The present study suggests that, under the model assumptions, combined use of two BCAs with different biocontrol mechanisms in most cases only results in control efficacies similar to using the more efficacious one alone. These predictions are consistent with published experimental results, suggesting that combined use of BCAs should not be recommended without clear understanding of their main biocontrol mechanisms and relative competitiveness, and experimental evaluation.

Additional keywords: coverage, modeling. we may also need to consider both direct and indirect interactions between different BCA populations. Use of mixtures of cultivars that differ in their resistance to diseases has been generally shown to reduce the rate of population increase of the pathogen (14, $27,33,34)$. Similarly, use of fungicide combinations with different modes of action has been recommended as a way to improve disease control, as well as to minimize the risk of development of resistance to fungicides $(4,42)$. There has been increasing interest in determining experimentally whether combined use (i.e., application of two BCA products simultaneously in a mixture or sequentially within a short interval) of two BCAs is more efficacious than use of individual BCAs $(6,7,15,18,39,45,48)$. Comparing with the more efficacious BCA of the two, combined use of two BCAs may lead to increased $(29,48)$, reduced $(3,11,45)$, or similar $(7,20,45)$ biocontrol efficacy; these outcomes have recently been interpreted in terms of the pathogen refuge size (22). In addition, a study using detached strawberry leaves demonstrated that variability in the biocontrol efficacy was reduced when two BCAs were applied together (18). The relative importance of individual mechanisms is often unknown in many of these experimental studies.

A previous numerical study showed that biocontrol efficacy was greatest for a single BCA combining competition with mycoparasitism or antibiosis (46). In this study, the generic model (21) was modified to take into account the circumstances of inundative application of BCA. Here, we take the investigation further to study the combined use of two BCAs with different biocontrol mechanisms with or without interactions between BCAs and where the two BCAs occupy the same tissue type as the pathogen. 
Therefore, the focus of this investigation was to determine whether combined use of two BCAs, each with a single but different mechanism, is more efficacious than use of individual BCAs or a single BCA with both biocontrol mechanisms. The disease dynamics were compared among the following scenarios: application of individual BCAs (each with a single mechanism) alone, combined use of two BCAs (each with a single but different mechanism); and a single BCA with both biocontrol mechanisms that are present separately in the two BCAs, as studied previously (46).

\section{THEORY AND APPROACHES}

Model description. The present model was based on the generic model developed by Jeger et al. (21), as modified for inundative application by $\mathrm{Xu}$ et al. (46). In this generic model (Fig. 1), a standard susceptible-infected-removed (SIR) model for host-pathogen dynamics was coupled with one for the pathogenBCA dynamics without considering spatial heterogeneity. Host tissue was divided into the following mutually exclusive categories: healthy susceptible $\left(H_{s}\right)$, healthy resistant to the pathogen $\left(H_{r}\right)$, infectious disease $(I)$, removed disease (postinfectious) $(R)$, and area occupied by BCA $\left(H_{b}\right)$. These state variables were defined in the linked differential equations. In the present model, $H_{r}$ and $H_{b}$ are subdivided into two mutually exclusive parts, indicated by subscript 1 or 2 . Thus, $H_{b 1}$ is the host tissue area colonized by BCA1, $H_{b 2}$ the area colonized by BCA2, $H_{r 1}$ is the host tissue area immune to colonization by BCA1, and $H_{r 2}$ the area immune to colonization by BCA2. The model assumes that two BCAs occupy the same type of host tissues as the pathogen. The full model in the case where there are no interactions between the two BCAs is then described in equation 1 .

The seven state variables add to 1.0. Descriptions for each model parameter are given in Table 1 . Here, subscripts 1 and 2 refer to BCA1 and BCA2, respectively. For the colonization rates, $c_{11}$ refers to the colonization rate of healthy host tissue $H_{s}$ by $\mathrm{BCA} 1$ and $c_{12}$ to that by $\mathrm{BCA} 2 ; c_{21}$ refers to the colonization rate of diseased host tissue $I$ by BCA1 and $c_{22}$ to that by BCA2. Dead BCA occupied tissues (the mortality is parameterized as $f$ ) to susceptible; this is used to simulate host growth.

Thus, in the model for two BCAs, the rate equations for $H_{r}$ and $H_{b}$ in $\mathrm{Xu}$ et al. (46, equations $2 \mathrm{~b}$ and c) are replaced by separate equations for $H_{r 1}, H_{r 2}$ and $H_{b 1}, H_{b 2}$. Similarly, where there is a term involving $H_{r}$ and $H_{b}$ in the equations 2 a to $\mathrm{d}$ in $\mathrm{Xu}$ et al. (46), they are replaced by the terms $H_{r 1}, H_{r 2}$ and $H_{b 1}, H_{b 2}$, together with their associated colonization rates as above. Comparing the model equations term-by-term with those in $\mathrm{Xu}$ et al. (46), equation 1a replaces equation $2 \mathrm{a}$; equations $1 \mathrm{~b}, 1 \mathrm{c}$ replace equation $2 \mathrm{~b}$; equations $1 \mathrm{~d}, 1 \mathrm{e}$ replace equation $2 \mathrm{c}$; and equation $1 \mathrm{f}$ replaces equation $2 \mathrm{c}$. Equation $1 \mathrm{~g}$ is redundant but is included for completeness to show that the rate equations add to zero (i.e., host tissue is of constant size). All assumptions in the case of no interactions between two BCAs remain as in Jeger et al. (21) with the modification introduced by $\mathrm{Xu}$ et al. (46) for inundative application of BCA.

Analytical analysis of the steady state and model behavior around the steady states was not possible because the model complexity does not lend itself to such analytical treatment. Thus, we determined the invasion criteria for only a few simplified cases and then carried out numerical simulations of the model to assess the general dynamics for combinations of some specific parameter values.

Conditions for disease or BCA initial invasion and establishment. Boundary conditions are defined as steady-state values in the absence of one or more of the host categories. We then determined criteria (conditions in terms of model parameter values) for invasion of the previously absent category. The boundary conditions are denoted by $\hat{H}_{s}, \hat{H}_{r 1}, \hat{H}_{b 1}, \hat{H}_{r 2}, \hat{H}_{b 2}$, and $\hat{I}$. There are three cases to consider.

Case 1: no disease and no BCAs $\left(\hat{H}_{s}=1\right)$. Pathogen will invade if $b / h>1$. Similarly, BCA1 will invade if $c_{11} / f_{1}>1$ and BCA2 will invade if $c_{12} / f_{2}>1$.

Case 2: no BCAs $\left(\hat{H}_{s}>0, \hat{I}>0\right)$. BCA1 will invade if $\hat{H}_{s}>\frac{f_{1}-c_{11}}{c_{11}-c_{12}}$ and BCA2 will invade if $\hat{H}_{s}>\frac{J_{2}-c_{22}}{c_{12}-c_{22}}$.

Case 3: no disease $\left(\hat{H}_{s}>0, \hat{H}_{r 1}>0, \hat{H}_{b 1}>0, \hat{H}_{r 2}>0, \hat{H}_{b 2}>0\right)$. Pathogen will invade if $b \hat{H}_{s}-c_{21} \hat{H}_{b 1}-c_{22} \hat{H}_{b 2}-h>0$ (i.e., the rate of increase in the infectious diseased area is greater than the total rate of loss in the infectious area, due to colonization by two BCAs and moving to removed diseased category). The steadystate values are obtained from simultaneous equations by setting the rate of change in the equations 1 a to e to zero (assuming $I=$ $0)$. However, there are potentially infinite solutions from the resulting simultaneous equations.

Numerical studies. In all cases where two BCAs were used (i.e., combined use), they were assumed to be introduced within a short interval and could be effectively considered to be introduced

$$
\begin{aligned}
& \frac{d H_{s}}{d t}=-b I H_{s}-\left(c_{11}+d_{1}\right) H_{b 1} H_{s}-\left(c_{12}+d_{2}\right) H_{b 2} H_{s}+f_{1} H_{b 1}+f_{2} H_{b 2}+e_{1} H_{r 1}+e_{2} H_{r 2} \\
& \frac{d H_{r 1}}{d t}=H_{b 1}\left(d_{1} H_{s}-c_{11} H_{r 1}\right)-c_{12} H_{b 2} H_{r 1}-e_{1} H_{r 1} \\
& \frac{d H_{r 2}}{d t}=H_{b 2}\left(d_{2} H_{s}-c_{12} H_{r 2}\right)-c_{11} H_{b 1} H_{r 2}-e_{2} H_{r 2} \\
& \frac{d H_{b 1}}{d t}=H_{b 1}\left[c_{11}\left(H_{s}+H_{r 1}+H_{r 2}\right)+c_{21}\left(1-H_{s}-H_{r 1}-H_{r 2}-H_{b 1}-H_{b 2}\right)-f_{1}\right] \\
& \frac{d H_{b 2}}{d t}=H_{b 2}\left[c_{12}\left(H_{s}+H_{r 1}+H_{r 2}\right)+c_{22}\left(1-H_{s}-H_{r 1}-H_{r 2}-H_{b 1}-H_{b 2}\right)-f_{2}\right] \\
& \frac{d I}{d t}=I\left(b H_{s}-c_{21} H_{b 1}-c_{22} H_{b 2}-h\right) \\
& \frac{d R}{d t}=h I-c_{21} R H_{b 1}-c_{22} R H_{b 2}
\end{aligned}
$$


at the same time for the present modeling study. Their order of introduction was not considered in the present study. Numerical simulation (by the Modified Extended Backward Differentiation Equation Implicit method) was carried out in Maple 11. Only a single set of parameters was used for the pathogen: $b=0.2$ and $h=0.05$; under this set of parameter values, at the absence of a BCA, the total disease reached $90 \%$ on day 70 . Numerical studies were divided into two parts: evaluating the model with or without interactions between two BCAs under three different BCA application strategies.

BCA application strategies. The effect of BCA coverage (50 to $90 \%$ ) on biocontrol efficacy was previously shown to be relatively small compared with other factors (46). Coverage is defined as the percentage of host tissues available for colonization by the BCAs that can be colonized by BCAs which are actually colonized following an application. There have been no experimental results on the colonization of host tissues by BCAs following an application. Thus, we did not consider coverage in this study but only investigated three application strategies that simulate three practical situations: (i) natural inoculum (i.e., no artificial introduction), (ii) application of BCAs at the onset of epidemic development, and (iii) application of BCAs in response to epidemic development. When an application of BCAs was made, the coverage was assumed to be $50 \%$.

Strategy 1. BCA was assumed to be present at the level of 0.001 , the same level as the infectious disease $(I)$; all the remaining host tissue belongs to the healthy susceptible category.

Strategy 2. As for strategy 1 , the initial disease was set to 0.001 infectious disease. The coverage of a single BCA application was assumed to be $50 \%$ and, hence, the total coverage of combined use (i.e., sequential applications within a short interval) of two BCAs under the assumption of independent with overlapping coverage was estimated to be $75 \%(=50 \%+[100 \%-$ $50 \%$ ] $\times 50 \%$ ). Thus, $37.5 \%$ of 0.999 healthy susceptible host tissue was allocated to each of the two BCAs.

Strategy 3. A BCA was applied at $50 \%$ coverage when the infectious disease just exceeded 0.01 (total disease [ $=$ infectious + postinfectious] was $\approx 0.0134$ at the time). If the main biocontrol mechanism of a BCA does not include antibiosis or mycoparasitism, only $50 \%$ of 0.9866 healthy susceptible tissue was

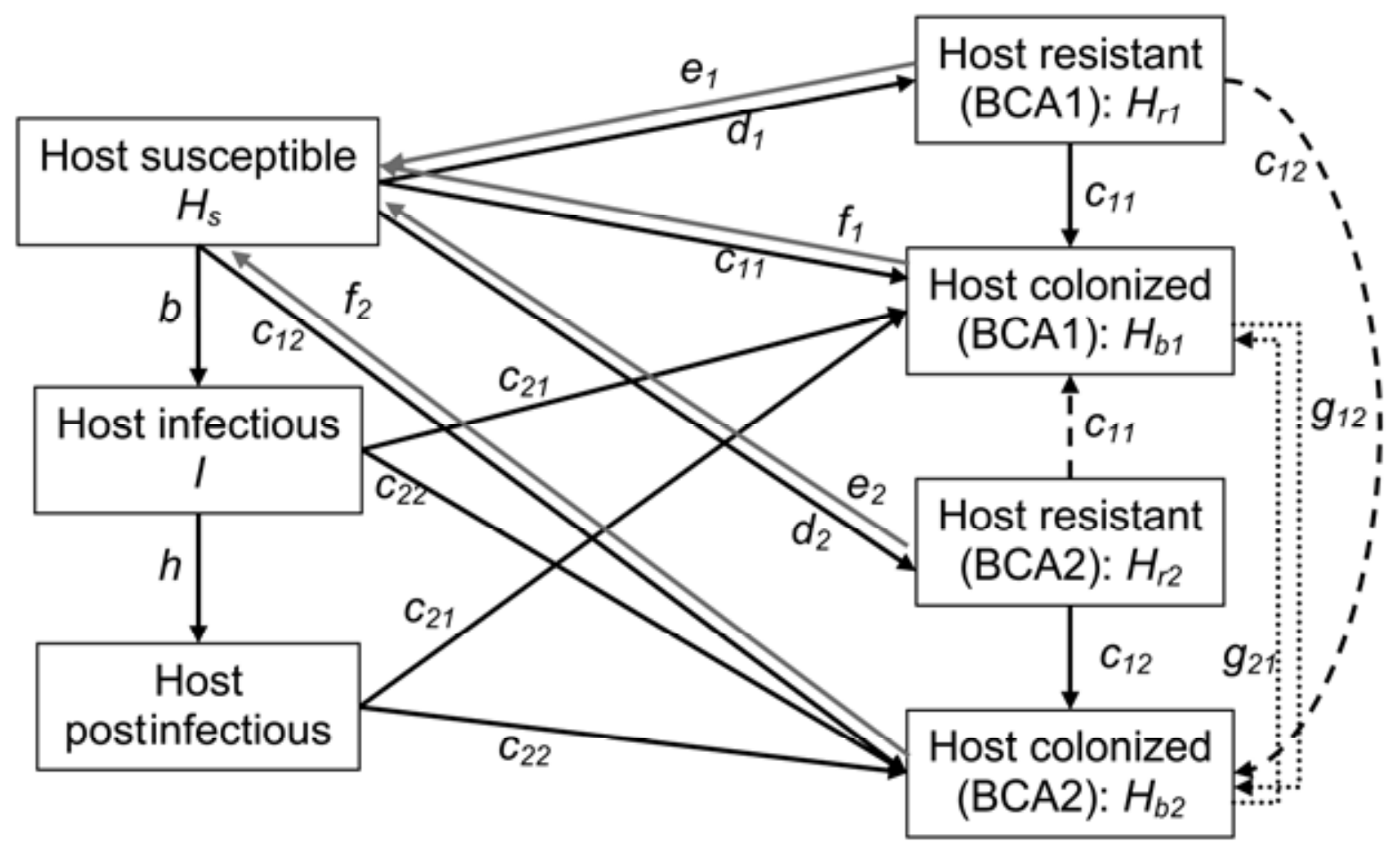

Fig. 1. Simplified flow diagram indicating the relationships among the host categories together with the rate parameters, describing the interrelationships among host, pathogen, and two biocontrol agents (BCAs). Dashed line and dotted arrows indicate indirect and direct interactions between the two BCAs, respectively.

TABLE 1. Default values of model parameters describing rates of changes of several processes in a plant pathogen biocontrol system; each set of parameter values represents a biocontrol agent (BCA) with a single main biocontrol mechanism as specified

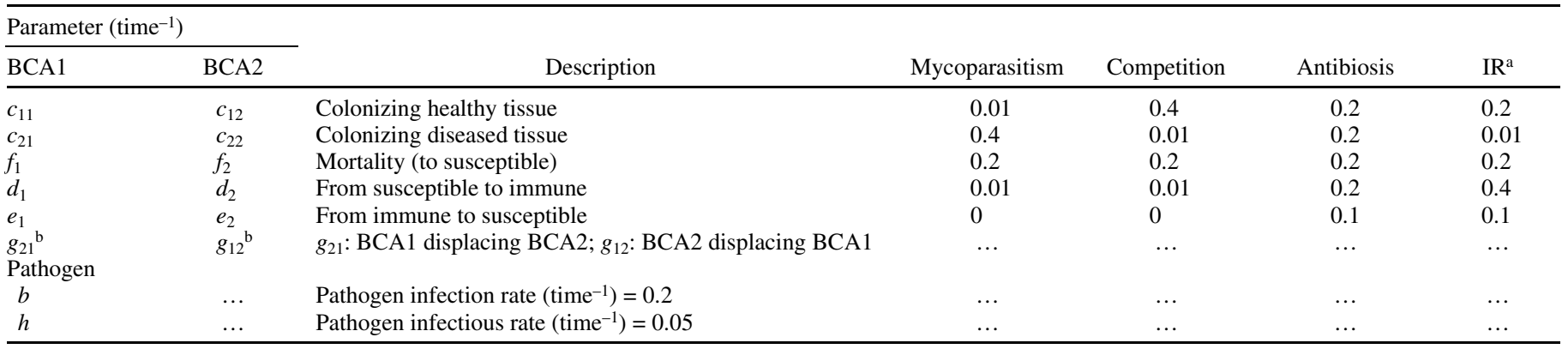

${ }^{a}$ Induced resistance.

$\mathrm{b}$ These two parameters are only used in the model that incorporates direct interactions between two BCAs; their exact values depend on the combination of two BCAs (text provides more details). 
allocated to the BCA when a single BCA was applied or $37.5 \%$ of 0.9866 healthy susceptible tissue to each BCA when two BCAs were used. However, with application of a single BCA whose main biocontrol mechanism included antibiosis or mycoparasitism, $50 \%$ of all types of host tissues, including infectious and removed disease, was allocated to the BCA because diseased tissues can also been colonized by a mycoparasitic or an antibiotic-producing BCA. Similarly, when two BCAs were applied, $37.5 \%$ of all host tissues was allocated to the mycoparasitic or antibiotic-producing $\mathrm{BCA}$; if one of the biocontrol mechanisms of the BCAs was the IR or competition, this BCA would only be allocated with $37.5 \%$ of the 0.9866 host tissues. The amount of infectious and removed disease was appropriately adjusted.

Without BCA interactions. We first conducted numerical evaluations to investigate the biocontrol efficacy of the combined use of two BCAs relative to a single $\mathrm{BCA}$ without considering interactions between BCAs (i.e., equation 1). Each biocontrol mechanism is characterized by one or more model parameters under the current model formulation: competition by higher $c_{11}$ (or $c_{12}$ ), mycoparasitism by higher $c_{21}$ (or $c_{22}$ ), antibiosis by average $c_{11}$ (or $c_{12}$ ) and $c_{21}$ (or $c_{22}$ ), and IR by higher $d_{1}$ (or $d_{2}$ ). Default parameter values defined for a $\mathrm{BCA}$ with a single main mechanism were the same as those reported previously (46) and given in Table 1. A BCA with two main biocontrol mechanisms was defined by combining the larger values of model parameters for each individual mechanism.

For each parameter that defines a biocontrol mechanism, a total of 21 different values were evaluated. Thus, $c_{11}$ or $c_{12}$ (competition), $c_{21}$ or $c_{22}$ (mycoparasitism), and $d_{1}$ or $d_{2}$ (IR) ranged from 0.2 to 0.6 in increments of 0.02 . Similarly, for antibiosis, either $c_{21}$ (or $c_{22}$ ) or $d_{1}$ (or $d_{2}$ ) varied from 0.05 to 0.35 in increments of 0.015 . When an antibiotic-producing BCA was used with either a competitive or an IR BCA, it was the $c_{21}$ (or $c_{22}$ ) that varied; when co-used with a mycoparasitic BCA, it was the $d_{1}$ (or $d_{2}$ ) that varied. All other parameters were set to the default values (Table 1); hence. this study did not consider possible interactions among two or more model parameters in affecting epidemic development. For a BCA that combined two mechanisms, the value of each relevant parameter defining a single mechanism was given the same value as that in the BCA with only the same single mechanism.

For each simulated disease dynamic, area under the disease progress curve (AUDPC) was calculated for total disease. Biocontrol efficacy was then estimated as $E_{b}=\left[\left(A U D P C-A U D P C_{B}\right)\right.$ $\times 100] / A U D P C_{P}$, where $A U D P C_{P}$ and $A U D P C_{B}$ are the AUDPC values for the simulated epidemics without and with BCA application, respectively. The model (equation 1) was evaluated for 350 days at a daily integration step because preliminary simulations suggested that, for most combinations of parameters, the system is approaching the steady-state by this time. Thus, differences in AUDPC values are likely to reflect more in the differences in the early stage of epidemic development between simulated epidemics.

With BCA interactions. Both direct and indirect interactions between two BCAs were investigated for their effects on epidemic development (Fig. 1). For indirect interactions, we assumed that BCA1 cannot colonize $H_{r 2}$, the area immune due to the presence of $\mathrm{BCA} 2$, and $\mathrm{BCA} 2$ cannot colonize $H_{r l}$, the area immune due to the presence of BCA1; in other words, the IR by one BCA is not only effective against the pathogen but also against the other BCA. Under this assumption, the $c_{12} H_{b 2} H_{r 1}$ and $c_{11} H_{b 1} H_{r 2}$ terms were omitted from equations $1 \mathrm{~b}$ and $1 \mathrm{~d}$, and $1 \mathrm{c}$ and 1e, respectively. For direct interactions between two BCAs, we assumed that BCA1 can displace BCA2 at a rate $g_{21}$ (note the reverse order of the labeling in the subscript) and BCA2 can displace BCA1 at a rate $g_{12}$. Thus, the term $\left(g_{21}-g_{12}\right) H_{b 1} H_{b 2}$ and $\left(g_{12}-g_{21}\right) H_{b 1} H_{b 2}$ was added to equations $1 \mathrm{~d}$ and $1 \mathrm{e}$, respectively.
Therefore, the magnitude and direction of the direct interaction depends on the joint presence of both BCAs (the product $H_{b 1} H_{b 2}$ ) and the sign of the difference $g_{21}-g_{12}$. Direct or indirect interactions were considered separately as well as jointly. In all cases, a BCA with a greater rate of colonizing healthy tissue was assumed to have greater competitiveness over the other BCA. An antibiotic-producing $\mathrm{BCA}$ was assumed to have greater competitiveness than a BCA providing IR.

Unless stated otherwise, $g_{21}$ and $g_{12}$ were assumed to be 0.2 and 0.0 , respectively. The effect of varying $g_{21}$ magnitude as well as the sign (i.e., switching the competitiveness between the two BCAs) was also investigated for several pairs of BCAs, one of which was either mycoparasitic or antibiotic producing. In order to study the long-term dynamics, the model was evaluated for 500 days at a daily integration step.

\section{RESULTS}

Without BCA interactions. As shown in previous studies (46), when a mycoparasitic BCA was used alone or in combination with another BCA, disease development exhibited greater oscillations, and this was also observed, to a lesser extent, for an antibiotic-producing BCA. Examples of such oscillating epidemics under three application strategies are shown in Figure 2.

Application strategy 1 . When co-introduced with the pathogen at the level of 0.001 , a BCA with IR as its main mechanism did not result in noticeable reductions in disease development over the entire range (0.2 to 0.6$)$ of $d_{1}$ values evaluated (Fig. 3A). Similarly, for an antibiotic-producing BCA, varying $d_{1}$ from 0.05 to 0.35 also did not result in noticeable disease reductions (Fig. 3C). For a BCA with competition as its main mechanism, increasing $c_{11}$ led to a gradual increase in disease reduction: $E_{b}$ increased from $0.7 \%$ when $c_{11}=0.2$ to $66.9 \%$ when $c_{11}=0.6$ (Fig. $3 \mathrm{~B})$; the absolute rate of increase in biocontrol efficacy increased with $c_{11}$. Similarly, for a mycoparasitic BCA, $E_{b}$ increased with increasing $c_{21}$ once $c_{21}>0.25$ and reached $59.5 \%$ when $c_{21}=0.6$.

In all cases, a single BCA with two biocontrol mechanisms was more effective than a single BCA with one mechanism or combined use of two BCAs with a single but different mechanism (Fig. 3). A BCA with both mycoparasitism and competition mechanisms was the most effective over the range of parameter values evaluated (Fig. 3D). Over the entire range of parameter values investigated, the efficacy resulting from combined use of two BCAs was nearly identical to the more efficacious one of the two, except for the combined use of antibiotic-producing and competitive BCAs (Fig. 3E). For this latter combination, the efficacy was similar to the more efficacious one (competition) but was less than the antibiotic-producing BCA (more efficacious one) for high parameter values (Fig. 3E).

The shape and magnitude of the increase in biocontrol efficacy with increasing parameter values varied with biocontrol mechanisms. Of the six pairs of biocontrol mechanisms, the increase in disease suppression with increasing parameter values was most steep for a single BCA combining competition with another mechanism, especially with antibiosis - this happened when $c_{11}=$ 0.325 and $c_{21}=0.135$ (Fig. 3E). For a BCA with single mechanism, an IR BCA was the least effective.

Application strategy 2. When a BCA was applied at a coverage of $50 \%$ at the beginning, the overall pattern was similar to that when a BCA was co-introduced under strategy 1, except for the following differences. First, as expected, increasing coverage to $50 \%$ led to a greater degree of disease suppression (Fig. 3 versus Fig. 4). Second, the parameter value required to achieve a similar level of disease suppression was decreased. Finally, when a competitive BCA was used with either a mycoparasitic or an antibiotic-producing BCA, it resulted in greater disease suppression than a single BCA for large parameter values (Fig. 4D and E). 
Application strategy 3. The main pattern was similar to those observed for strategies 1 and 2. Disease suppression achieved in this strategy was between those achieved by strategies 1 and 2 (Figs. 3, 4, and 5).

With BCA interactions. With application strategy 1, neither indirect nor direct interactions between pairs of BCAs with different mechanisms had any discernable effect on the resulting disease development, except for the combined use of mycoparasitic and competitive BCAs. For this pair of BCAs, direct interactions (the competitive BCA over the mycoparasitic BCA) led to a shifted oscillation in the disease dynamics (Fig. 6).

In contrast to strategy 1 , disease development for strategy 2 was considerably affected by the type of interactions between two BCAs for all pairs of mechanisms except competition with either IR or antibiosis (Fig. 7). The effect of BCA interactions was more pronounced toward the mid- to late phase of epidemic development. In all cases except IR with antibiosis (Fig. 7A), there were no discernable differences in epidemic dynamics by introducing indirect interactions between two BCAs. However, directed interactions between BCAs led to considerable changes in disease development, particularly for cases involving a mycoparasitic BCA (Fig. 7D to F). When a mycoparasitic BCA was used with either an antibiotic-producing (Fig. 7D) or a competitive (Fig. 7F) BCA, the direct interaction increased the period length of the fluctuation in disease dynamics; in contrast, the combined use of IR and mycoparasitic BCAs shortened the period length (Fig. 7E). The overall effect of indirect or direct interaction on disease development was similar between strategies 2 and 3 .

Because only direct interactions appeared to have appreciable effects when a mycoparasitic BCA was used, further evaluation was conducted to investigate the effect of varying $g_{21}$ values. When a mycoparasitic BCA was assumed to be less competitive, increasing $g_{21}$ led to greater disease development initially when used with an either competitive or IR BCA (Fig. 8A and B). The value of 0.4 for $g_{21}$ led to errors in numerical integration for the combined use of mycoparasitic and competitive BCAs (Fig. 8A). However, when the mycoparasitic BCA was assumed to be more competitive (i.e., $g_{21}<0$ ), increasing the magnitude of $g_{21}$ led to less disease development as well as the fluctuation in disease dynamics when used with a competitive and, to a lesser extent, an antibiotic-producing BCA (Fig. 9A and C). The opposite was true for the combination of mycoparasitic and IR BCAs (Fig. 9B). Increasing competitiveness of an antibiotic-producing BCA over a competitive one led to reduced disease development after the initial phase (Fig. 10A). In contrast, increasing competitiveness of a competitive BCA over an antibiotic-producing one did not result in any appreciable differences in disease development (Fig. 10B).

\section{DISCUSSION}

A previous analytical study showed the importance of three biocontrol parameters in determining biocontrol outcomes-the rates of a BCA colonizing healthy and diseased host tissues and BCA mortality (21). Further numerical evaluation of a modified version of this analytical model suggested that biocontrol efficacy was greatest for a single BCA combining competition with either mycoparasitism or antibiosis (46). However, the present study suggests that combined use of two BCAs, each with a single main mechanism, generally would not result in appreciable improvement in biocontrol over individual BCAs applied alone. Furthermore, introduction of interactions between two BCAs did not result in any discernable effects on disease development when neither a mycoparasitic nor an antibiotic-producing BCA was involved.

Conclusions drawn from any numerical studies depend on precise parameter values used. Although the magnitudes of these values used in the present study are open to question due to the lack of published data to estimate them, the main conclusions regarding the combined use of BCAs are expected to stand because they are consistent over a wide range of values evaluated for each key parameter. Parameter values from the few published experimental studies fell into the range evaluated in this study. To the best of our knowledge, only a single study reported the rate of BCA colonization of healthy tissue; namely, colonization of necrotic cyclamen tissue by Ulocladium atrum, as a competitive BCA against Botrytis cinerea (24), in which the BCA colonization rate $\left(0.031\right.$ to $\left.0.056 \mathrm{~h}^{-1}\right)$ is greater than the value used here. However, the $U$. atrum rate was estimated for a logistic model and it would be smaller when adjusted for the upper asymptote. Furthermore, the overall rate of colonization by U. atrum was less than that of B. cinerea. Similarly, the rate of the BCA colonizing diseased tissue was slightly less than a single reported value (30). Thus, the present study may have overestimated biocontrol efficacy for both competitive and mycoparasitic BCAs. The mortality value of 0.2 used in the present study is within the range of observations of several BCAs $(2,12,13,49)$, as is the rate $(0.1)$
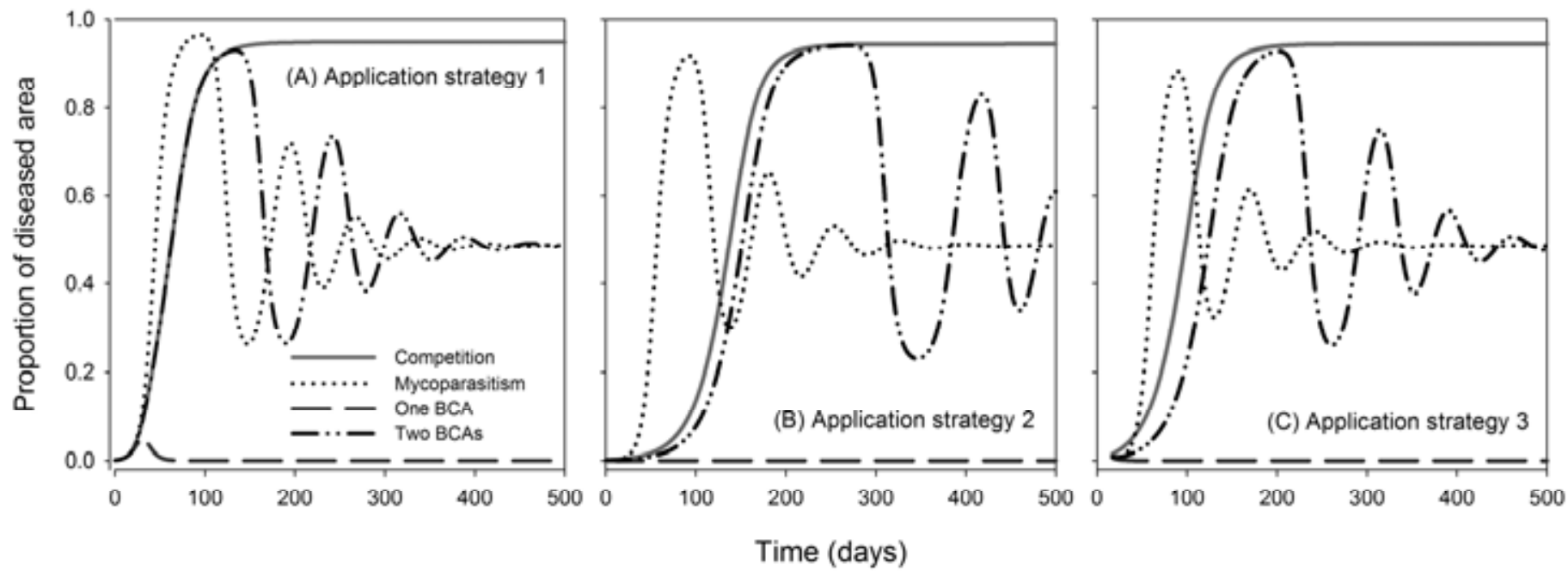

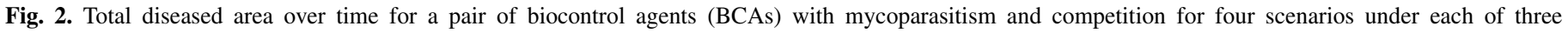

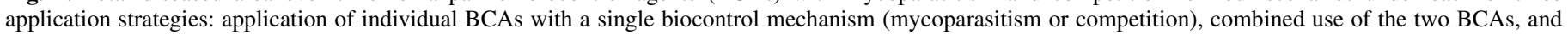

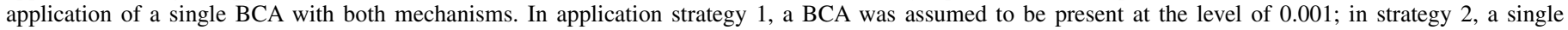

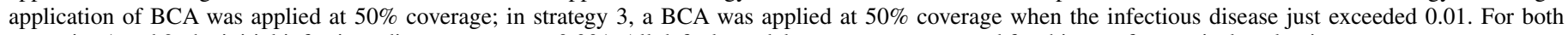
strategies 1 and 2, the initial infectious disease was set to 0.001. All default model parameters were used for this set of numerical evaluations. 
Rate of colonizing diseased tissue (antibiosis)
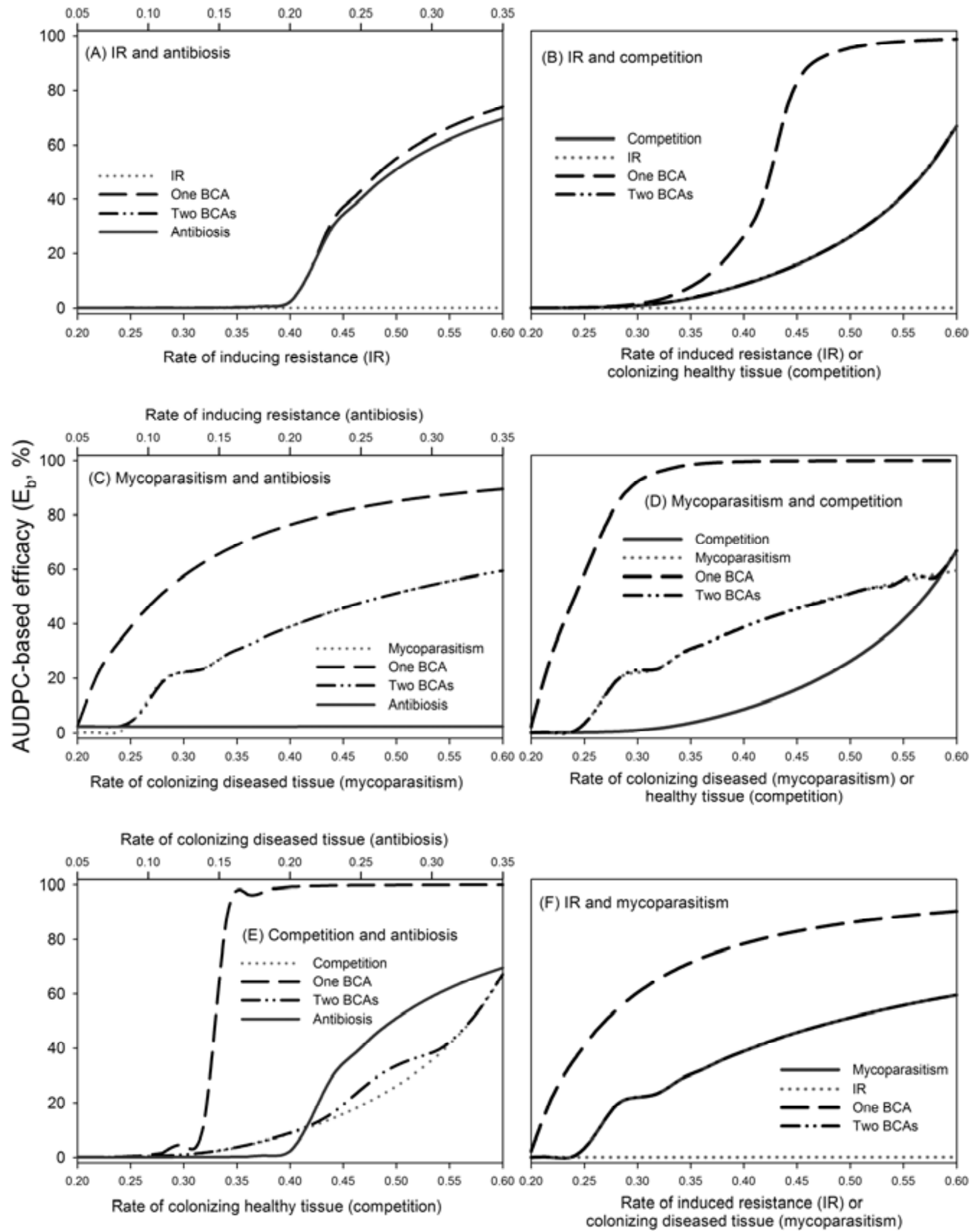

Fig. 3. Estimated biocontrol efficacy (\%) based on area under the disease progress curve values calculated from simulated epidemics for each pair of biocontrol agents (BCAs) with different combination of biocontrol mechanisms under the four scenarios for application strategy 1: application of individual BCAs with a single (but different) biocontrol mechanism as specified in the legend, combined use of these two BCAs, and application of a single BCA with both mechanisms. In this application strategy, the BCAs were introduced at 0.001 at the same time as the pathogen (infectious disease $I=0.001$ ). In total, 21 values were used for each key parameter defining a single biocontrol mechanism (see text). In each graph, if the range of the parameter value is the same for the two key parameters (one for each BCA), a single x-axis is used; otherwise, two x-axes (upper and low) are used. For all other parameters, default values were used. In many cases, the biocontrol efficacy for the combined use of two BCAs overlaps that of the more efficacious one of two component BCAs. 
Rate of colonizing diseased tissue (antibiosis)

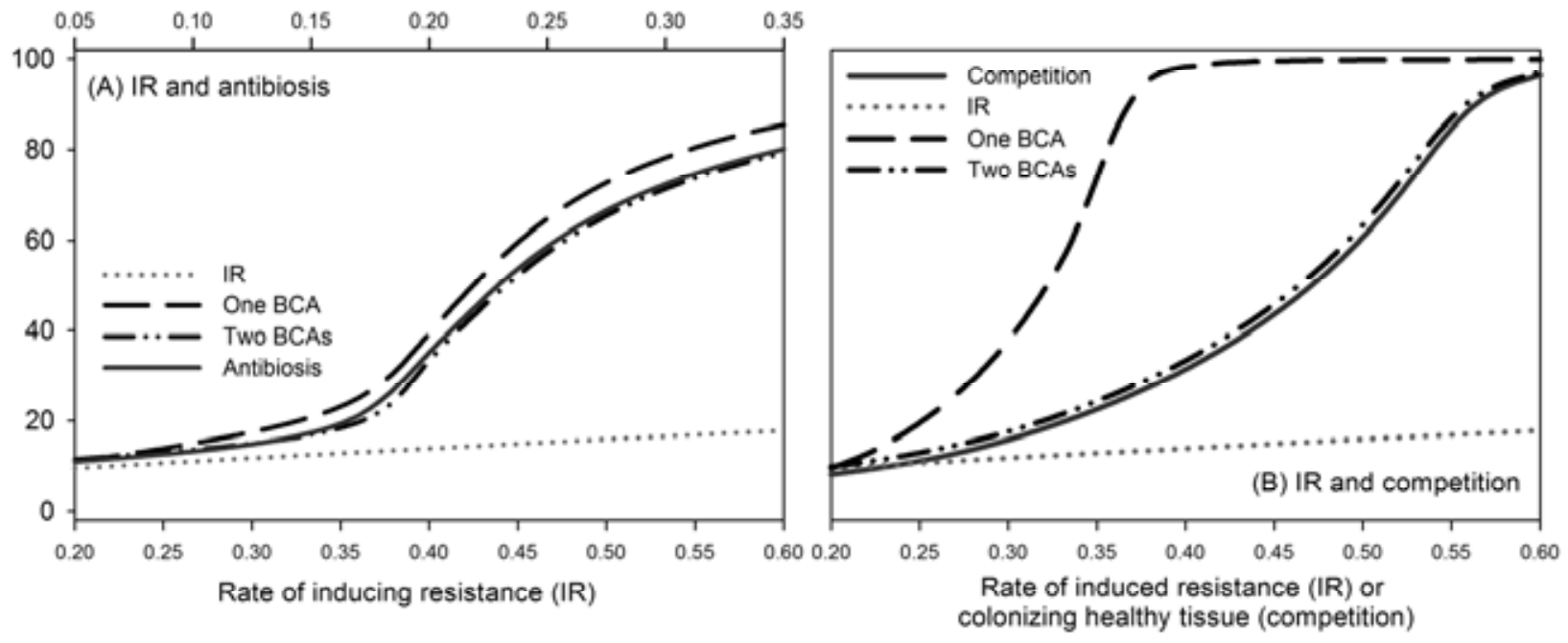

Rate of inducing resistance (antibiosis)

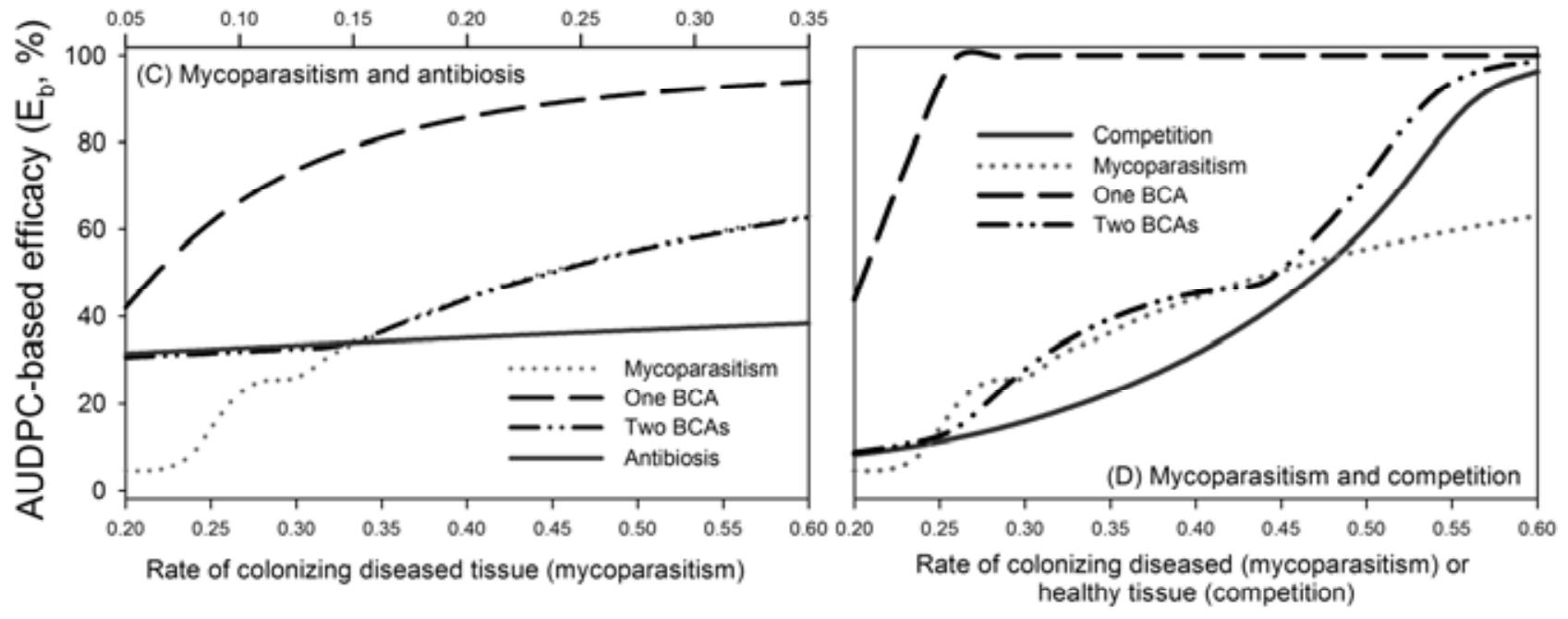

Rate of colonizing diseased tissue (antibiosis)

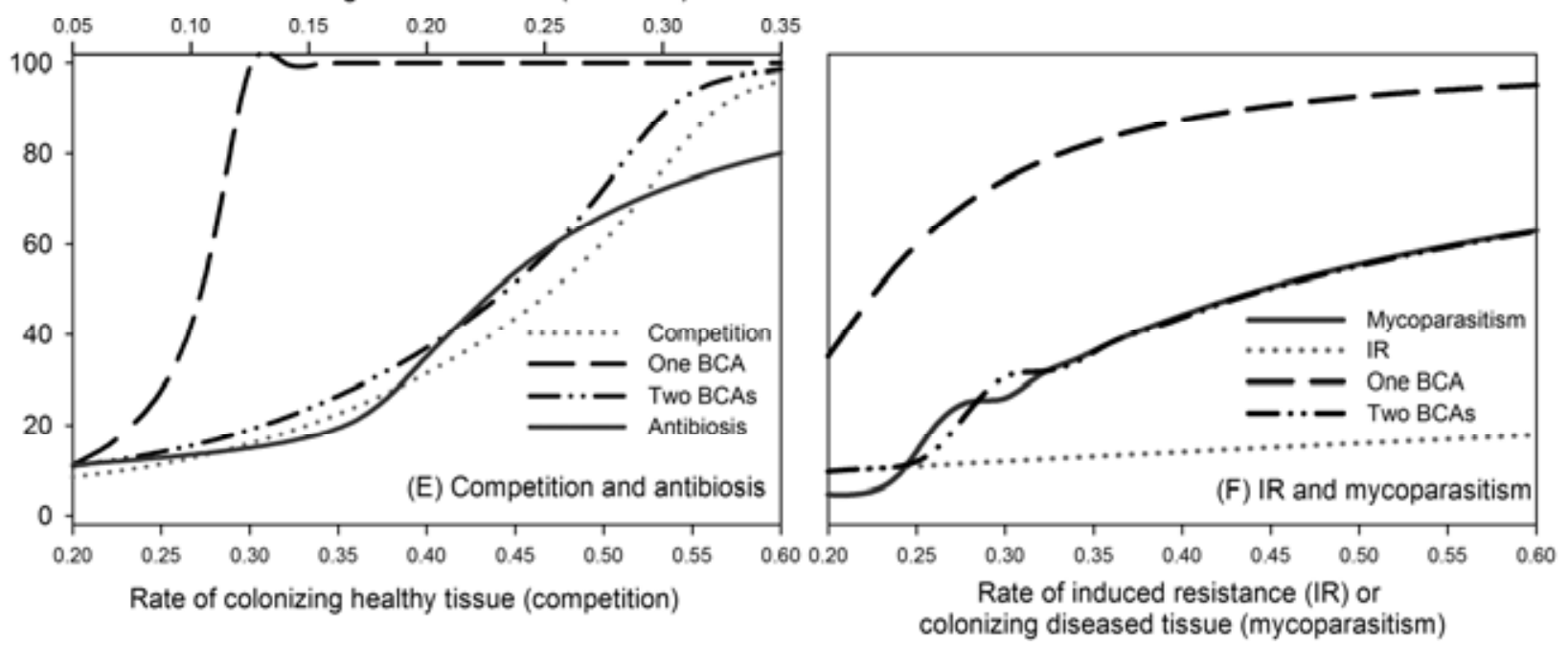

Fig. 4. Estimated biocontrol efficacy (\%) based on area under the disease progress curve values calculated from simulated epidemics for each pair of biocontrol agents (BCAs) with different combination of biocontrol mechanisms under the four scenarios for application strategy 2: application of individual BCAs with a single (but different) biocontrol mechanism as specified in the legend, combined use of these two BCAs, and application of a single BCA with both mechanisms. In this application strategy, the BCAs were applied at 50\% coverage at the same time as the pathogen (infectious disease $I=0.001$ ). In total, 21 values were used for each key parameter defining a single biocontrol mechanism (see text). In each graph, if the range of the parameter value is the same for the two key parameters (one for each BCA), a single x-axis is used; otherwise, two x-axes (upper and low) are used. For all other parameters, default values were used. In many cases, the biocontrol efficacy for the combined use of two BCAs overlaps that of the more efficacious one of two component BCAs. 


\section{Rate of colonizing diseased tissue (antibiosis)}

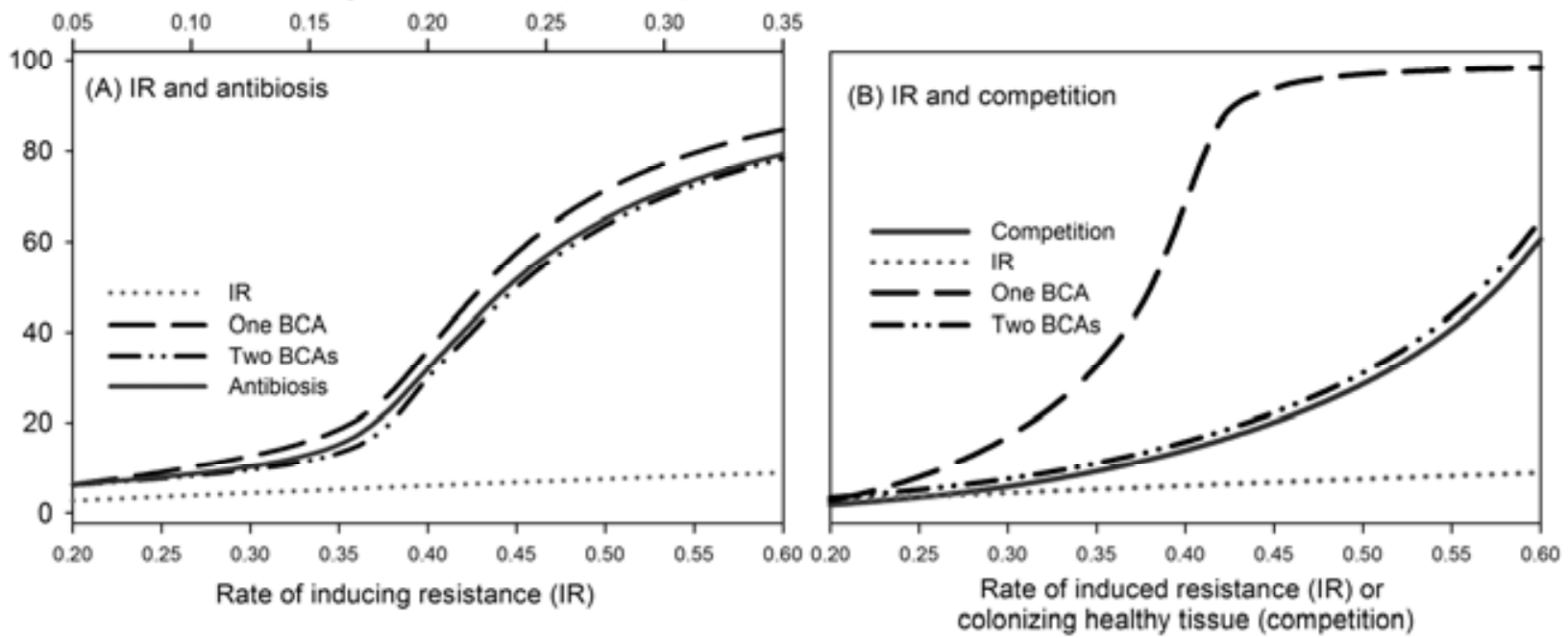

Rate of inducing resistance (antibiosis)

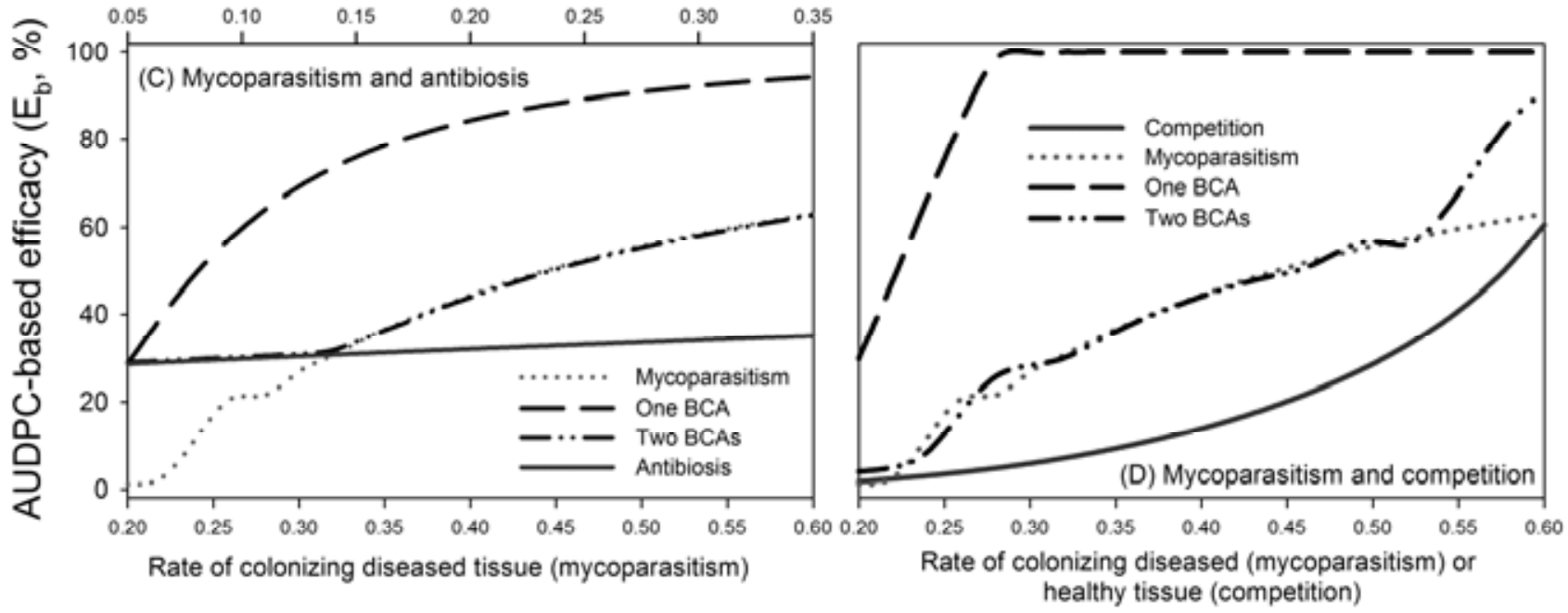

Rate of colonizing diseased tissue (antibiosis)

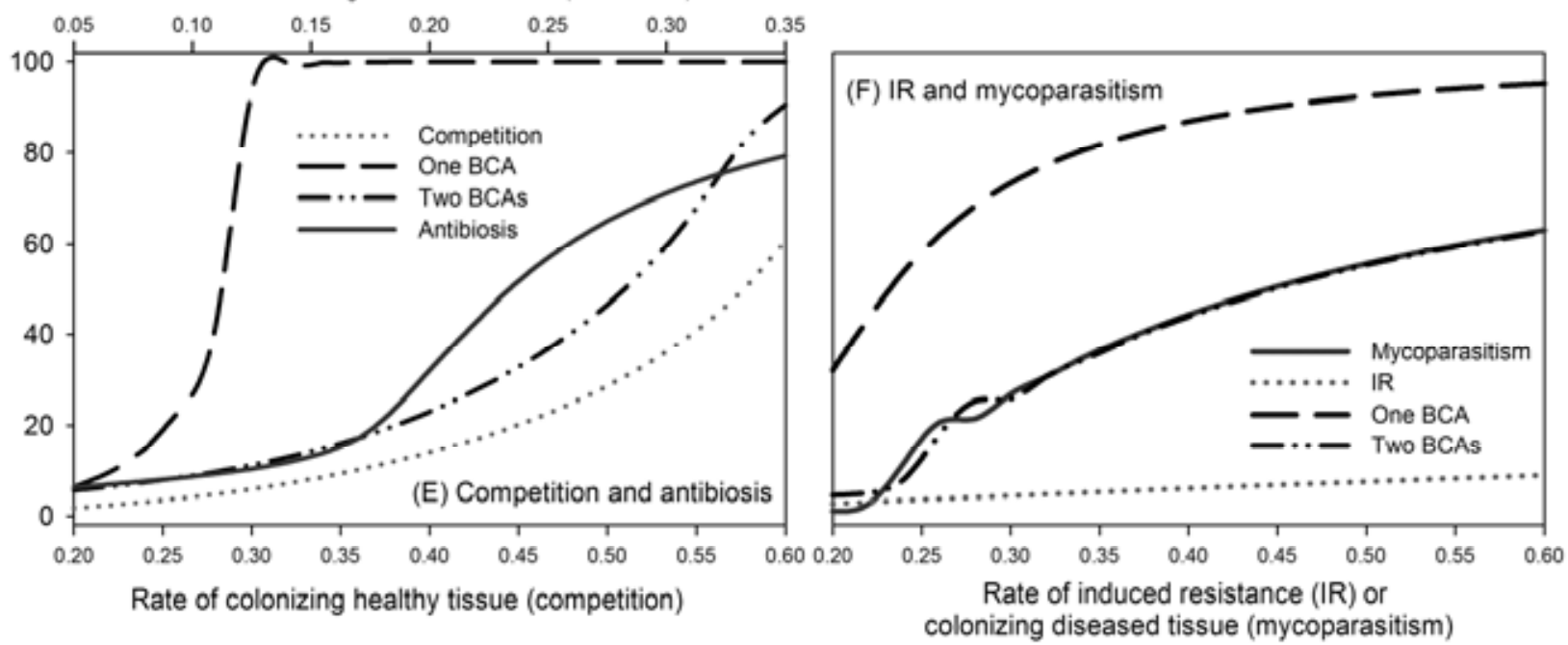

Fig. 5. Estimated biocontrol efficacy (\%) based on area under the disease progress curve values calculated from simulated epidemics for each pair of biocontrol agents (BCAs) with different combinations of biocontrol mechanisms under the four scenarios for application strategy 3: application of individual BCAs with a single (but different) biocontrol mechanism as specified in the legend, combined use of these two BCAs, and application of a single BCA with both mechanisms. In this application strategy, the BCAs were applied at 50\% coverage at the same time as the pathogen (infectious disease $I=0.01$ and removed disease $=0.0034$ ). In total, 21 values were used for each key parameter defining a single biocontrol mechanism (see text). In each graph, if the range of the parameter value is the same for the two key parameters (one for each BCA), a single x-axis is used; otherwise, two x-axes (upper and low) are used. For all other parameters, default values were used. In many cases, the biocontrol efficacy for the combined use of two BCAs overlaps that of the more efficacious one of two component BCAs. 
of loss of IR $(19,38)$. The most uncertain parameter values are associated with antibiosis, where we have been unable to find quantitative data. In many scenarios, biocontrol efficacy increased steeply with parameter values, indicating the importance of estimating these parameters under various environmental conditions for predicting biocontrol potentials. This study also suggested that a BCA with IR as its main mechanism is not likely to succeed unless it is repeatedly applied; and that, for a BCA with competition as its main mechanism to succeed, its rate of colonizing healthy tissue needs to be at least twice of the rate of pathogen colonization.

As shown in a previous study (46), a BCA combining two main biocontrol mechanisms, especially competition with either mycoparasitism or antibiosis, was much more effective in suppressing disease development than a BCA with a single main mechanism over the entire range of parameter values evaluated. However, combined use of two BCAs, each with a single but different mechanism, did not generally lead to increased disease suppression compared with use of individual BCAs alone and only achieved biocontrol efficacies nearly identical to those achieved by the more efficacious one. The lack of disease suppression by combined use of two BCAs, compared with the use of a single BCA with the same two mechanisms, can be explained by the fact that, unlike a single BCA with both mechanisms, the combined use of two BCAs failed to exploit the advantage of higher rates of colonizing healthy tissue in one BCA and diseased tissue in the other. Thus, combining two mechanisms in a single BCA may be more effective in reducing the size of pathogen refuge (22) than combined use of two BCAs, each with a single mechanism. In addition, the present model may have overestimated the efficacy for combined use of two BCAs because a total $75 \%$ coverage was assumed for the combined use of two BCAs compared with 50\% for using a single BCA alone. These results are generally consistent with experimental studies that demonstrated that combined use of two BCA products usually did not result in improved disease control over the more efficacious component (44). For example, combined use of Trichoderma and Bacillus isolates as a

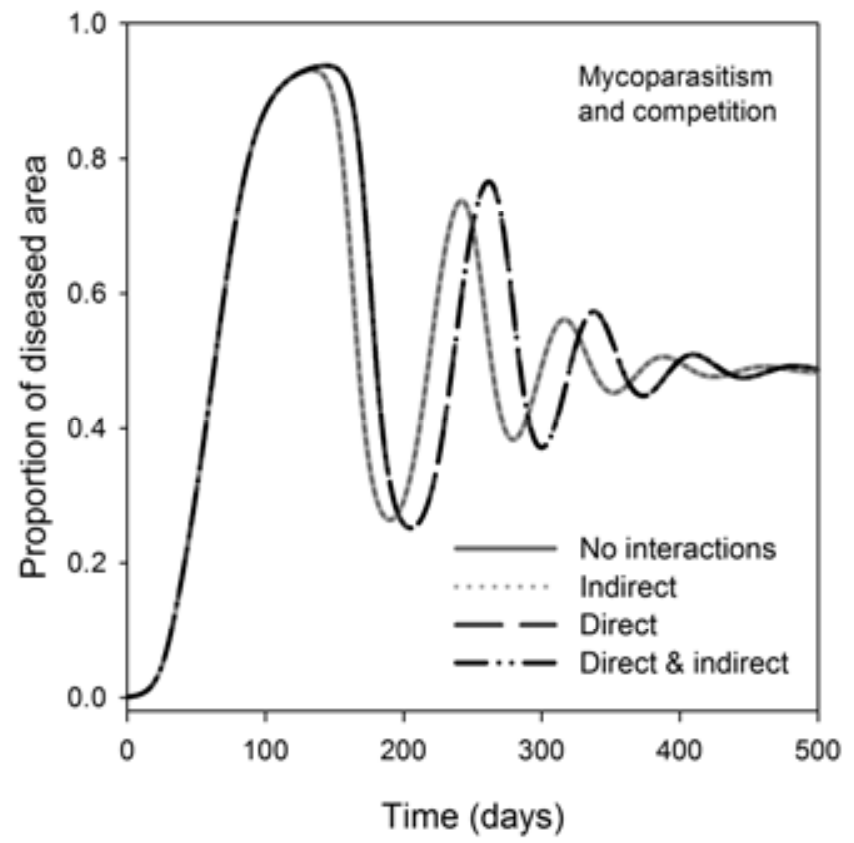

Fig. 6. Total diseased area over time when a mycoparasitic and a competitive biocontrol agent (BCA) were introduced at the same time as the pathogen at the level of 0.001 infectious disease (i.e., application strategy 1) with or without interactions between the two BCAs. For all other pairs of mechanisms, there were no discernable effects of BCA interactions on the disease dynamics. All default model parameters were used for this set of numerical evaluations. seed-coating treatment resulted in better control of damping-off of cucumber caused by Rhizoctonia solani than the use of individual isolates alone (48). On the other hand, combined use of Streptomyces lydicus with other BCAs did not result in as much in vitro inhibition of Phytophthora ramorum as achieved by individual BCAs (11). Combinations of BCAs taken directly from tomato leaves may improve gray mold control (17) but statistical analysis was not conducted to test the existence of synergistic effects between two BCAs. Subsequent research has suggested that there is no statistical evidence for synergy between the two BCAs (18).

Indirect interactions between two BCAs, as formulated in the present model, generally did not have any noticeable effects on suppressing disease development. Indirect interactions only relate to immune host tissues. Colonization of immune host tissues by a non-IR BCA is only expected to influence disease development if the mortality of this BCA is either much greater or much less than the rate of immune tissues reverting to the susceptible category. In contrast, direct interactions between two BCAs can greatly influence disease development, particularly when a mycoparasitic $\mathrm{BCA}$ is involved. In general, increasing competitiveness of a mycoparasitic BCA over either a competitive or an antibioticproducing BCA may increase biocontrol efficacy and dampen disease fluctuations. The opposite will be true when a mycoparasitic BCA is less competitive. Similar trends were also observed when an antibiotic-producing BCA was involved, although to a lesser extent. Reduced variability in biocontrol in combined use of two BCAs (one is believed to act via antibiotics and the other via competition) on strawberry leaf discs may partially result from such reduced fluctuations in disease dynamics due to interactions between two BCAs (17). Similar results were also obtained in biocontrol of Rhizoctonia damping-off and Fusarium wilt of tomato via combined use of BCAs (39). Whether such a phenomenon of reduced variability in biocontrol efficacy is common for combined use of BCAs needs further experimental and theoretical investigations.

This numerical study did not consider several other factors that may affect biocontrol efficacy when two BCAs are used. Homogeneous mixing between BCA and pathogen was assumed, although heterogeneous mixing is usually the case in practice $(16,22,31,32)$. Spatiotemporal variation in external conditions was not considered. It is well known that BCA, pathogen, and plant growth may be differentially affected by varying conditions. For example, the relative biocontrol performance of a single BCA and two BCAs varied greatly with the extent of temperature fluctuation (45). These spatiotemporal heterogeneities in driving variables will lead to corresponding spatial heterogeneities in model parameter values. However, as reasoned before, such heterogeneities are not likely to alter the two main conclusions: (i) combined use of two BCAs is generally not likely to result in increased efficacies compared with the more efficacious component and (ii) a single BCA with two different mechanisms is more efficacious than combined use of two BCAs, each with a single but different mechanism.

Spatial separation of BCAs on the host via colonization of separate host tissues such as roots, leaves, and flowers is expected to improve biocontrol efficacy because, in this situation, there is no direct or indirect interaction or competition between BCAs except via the pathogen. This is particularly the case when a pathogen is able to infect different host tissues, such as $B$. cinerea. The improved efficacy from combined use of two BCAs that occupy different niches was observed for $B$. cinerea on tomato $(28,29)$. However, the present model assumes that two BCAs control the same pathogen at the same infection court. In a similar concept, combined use of two BCAs may lead to a much reduced pathogen refuge size (22). The present study did not explicitly model the size of pathogen refuge size but determined long-term dynamics of pathogen development in a biocontrol 

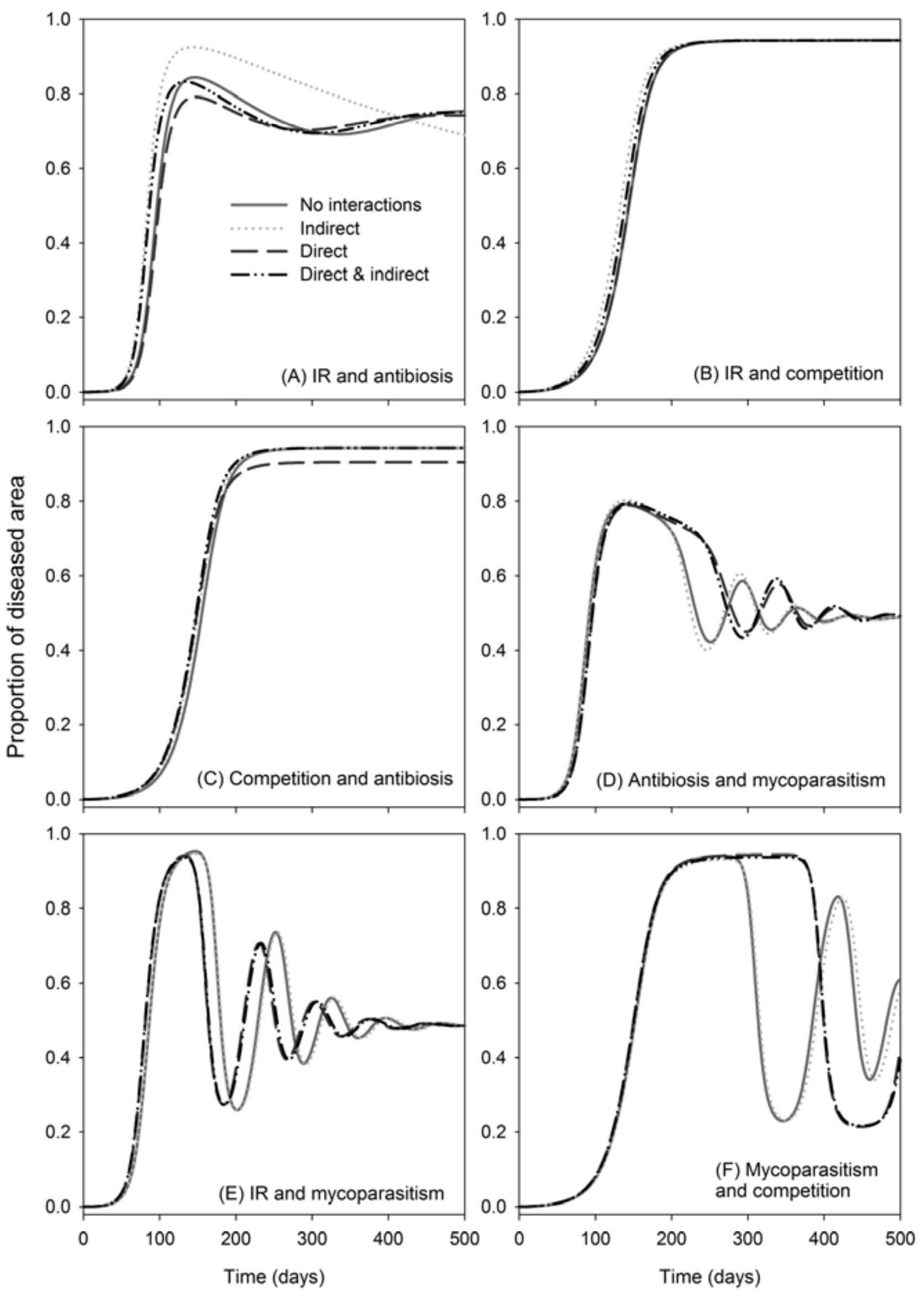

Fig. 7. Total diseased area over time when two biocontrol agents (BCAs), each with a single but different mechanism, were introduced at coverage of 50\% at the same time as the pathogen at the level of 0.001 infectious disease (i.e., application strategy 2) with or without interactions between the two BCAs. All default model parameters were used for this set of numerical evaluations. 

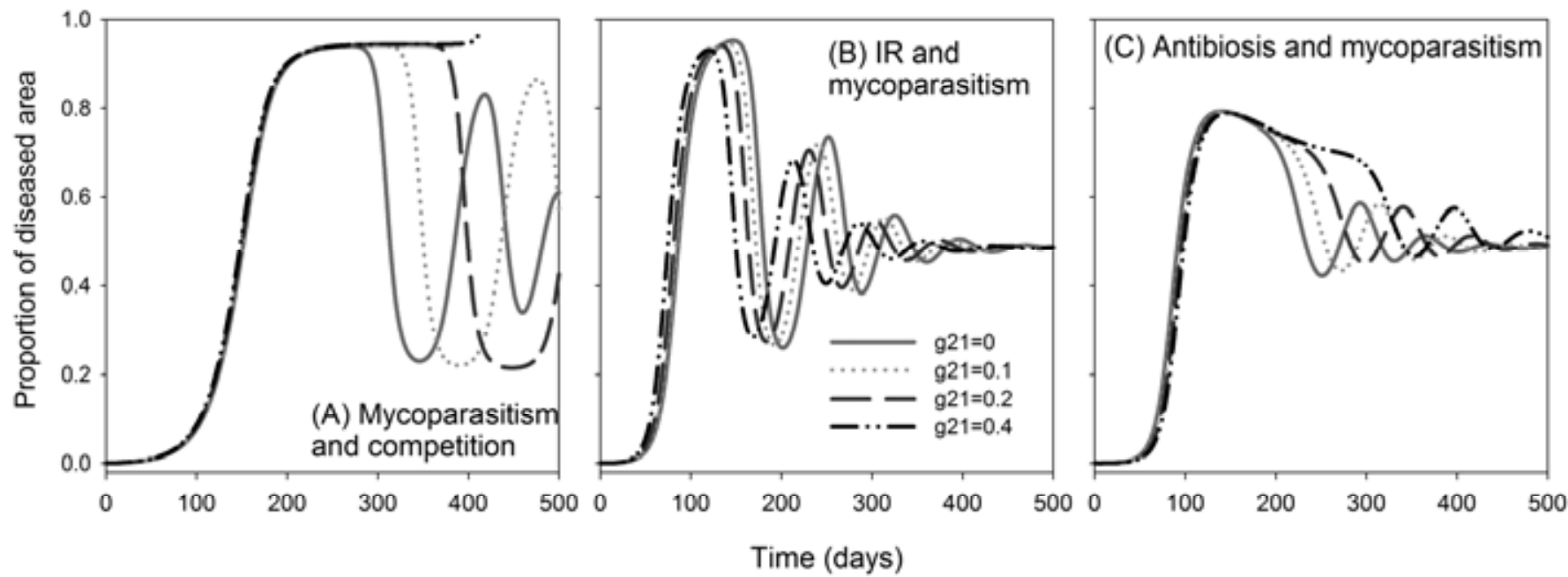

Fig. 8. Total diseased area over time when a mycoparasitic biocontrol agent (BCA), assumed to be less competitive, was introduced with another BCA at coverage of $50 \%$ at the same time as the pathogen at the level of 0.001 infectious disease (i.e., application strategy 2) with differing degrees of direct interactions between a mycoparasitic BCA and A, a competitive BCA; $\mathbf{B}$, an induced resistance (IR) BCA; or $\mathbf{C}$, an antibiotic-producing BCA, specified by the parameter $g_{21}$. For all other parameters, default values were used.
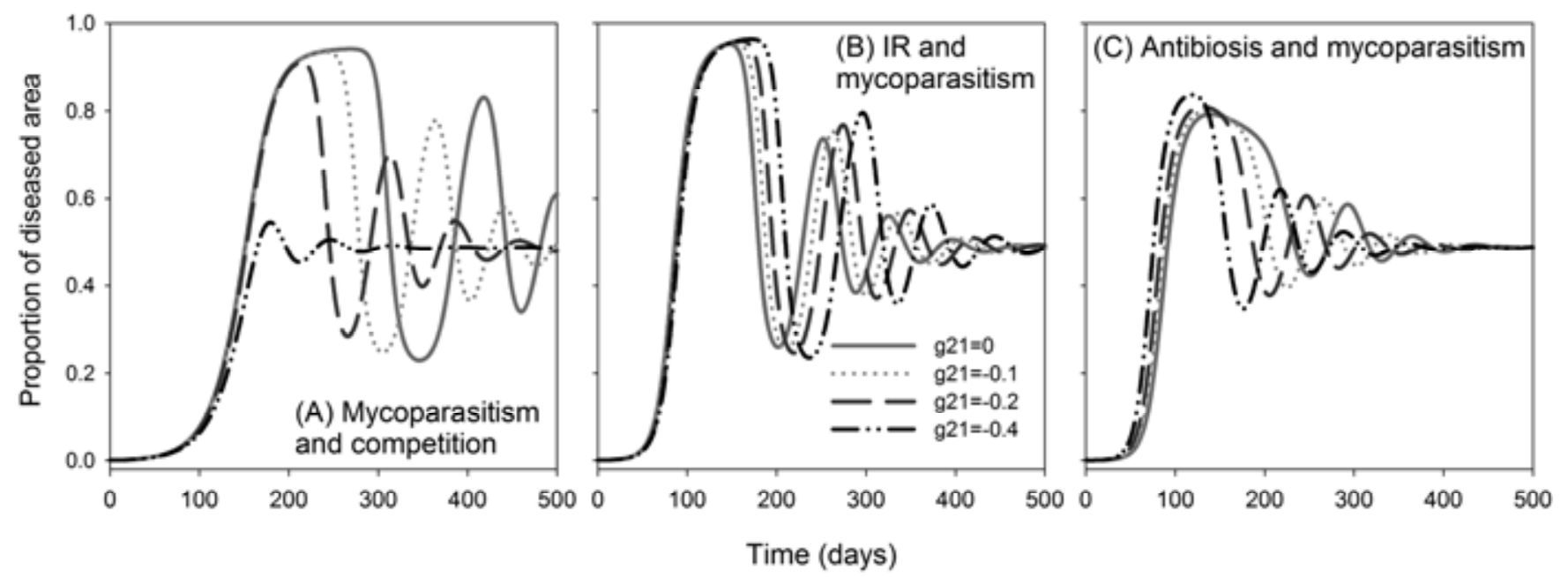

Fig. 9. Total diseased area over time when a mycoparasitic biocontrol agent (BCA), assumed to be more competitive, was introduced with another BCA at coverage of $50 \%$ at the same time as the pathogen at the level of 0.001 infectious disease (i.e., application strategy 2) with differing degrees of direct interactions between a mycoparasitic BCA and $\mathbf{A}$, a competitive BCA; B, an induced resistance (IR) BCA; or $\mathbf{C}$, an antibiotic-producing BCA, specified by the parameter $g_{21}$. For all other parameters, default values were used.
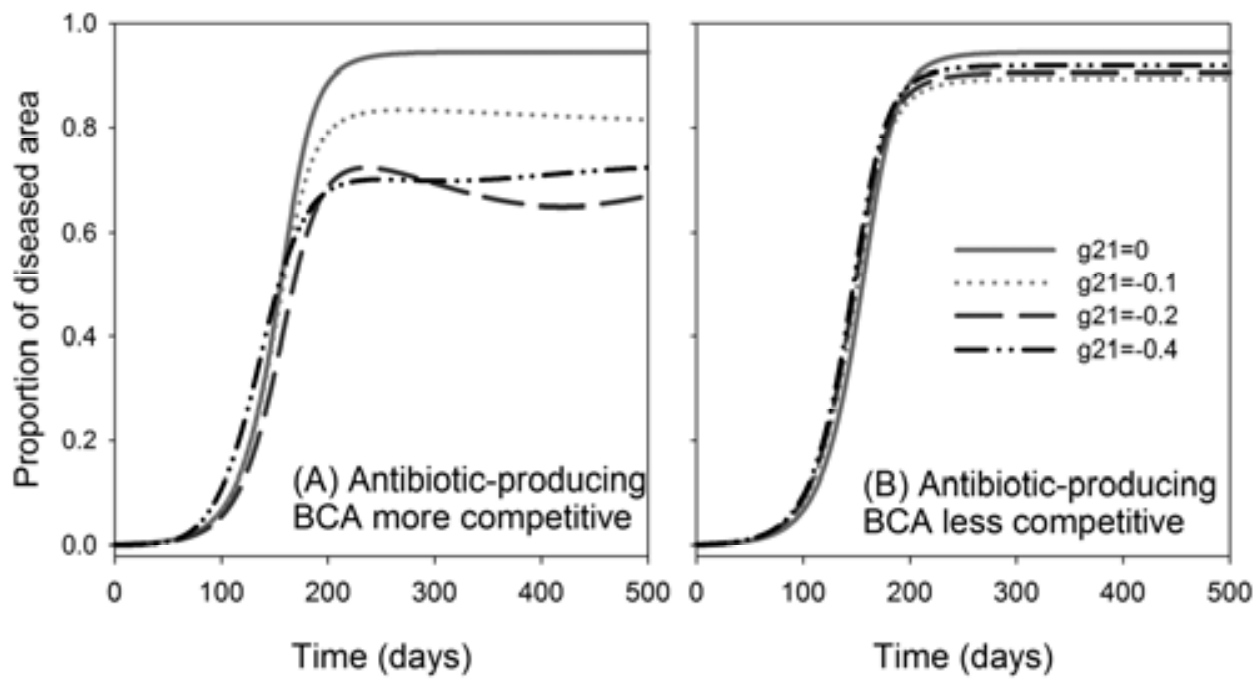

Fig. 10. Total diseased area over time when an antibiotic-producing biocontrol agent (BCA) was introduced with a competitive $\mathrm{BCA}$ at coverage of $50 \%$ at the same time as the pathogen at the level of 0.001 infectious disease (i.e., application strategy 2) with differing degrees of direct interactions between the two BCAs, specified by the parameter $g_{21}$ : the antibiotic-producing BCA is $\mathbf{A}$, more or $\mathbf{B}$, less competitive. For all other parameters, default values were used (Table 1). 
system where the pathogen steady-state value may be considered as the size of pathogen refuge under a given set of conditions. Only one factor studied here is directly related to the pathogen refuge size: spray coverage. Previous modeling results suggested a diminished return in biocontrol efficacy once the coverage is $>50 \%$ (46), which is consistent with the concept of pathogen refuge size (22). This study did not consider the scenario of sequential application of two BCAs over a longer time span $(37,40,45)$. In such a case, biocontrol efficacy may be influenced by application order (40) and, possibly, the time delay between application of the two BCAs.

In summary, this numerical study showed that the combined use of two BCAs, each with a single but different mechanism, does not generally result in appreciable improvement in biocontrol efficacy over individual BCAs applied alone. In some cases, combined use of two BCAs may increase as well as reduce biocontrol efficacy, depending on the type and extent of interactions between the two BCAs concerned. Thus, it is unlikely that combined use of two BCAs will lead to synergy in biocontrol, a conclusion which is supported by published experimental results thus far (44). Further specific experimental studies are needed to test this hypothesis. Therefore, combined use of BCAs should not be generally recommended in practice without clear understanding of their main biocontrol mechanisms and relative competitiveness.

\section{ACKNOWLEDGMENTS}

This research was funded by the U.K. Chemicals Regulation Directorate (CRD), Department of Environment Food and Rural Affairs (Defra) (project number 2119).

\section{LITERATURE CITED}

1. Arras, G, and Arru, S. 1997. Mechanism of action of some microbial antagonists against fungal pathogens. Ann. Microbiol. Enzimol. 47:97120.

2. Boff, P. 2001. Epidemiology and Biological Control of Grey Mould in Annual Strawberry Crops. Wageningen University, Wageningen, The Netherlands.

3. Bora, T., Ozaktan, H., Gore, E., and Aslan, E. 2004. Biological control of Fusarium oxysporum f. sp. melonis by wettable powder formulations of the two strains of Pseudomonas putida. J. Phytopathol. 152:471-475.

4. Brent, K. J., and Hollomon, D. W. 2007. Fungicide Resistance in Crop Pathogens: How Can It be Managed? 2nd ed. FRAC Monograph No. 1. Croplife International, Brussels.

5. Cabrefiga, J, and Montesinos, E. 2005. Analysis of aggressiveness of Erwinia amylovora using disease-dose and time relationships. Phytopathology 95:1430-1437.

6. de Boer, M., Born, P., Kindt, F., Keurentjes, J. J. B., van der Sluis, I., van Loon, L. C., and Bakker, Pahm. 2003. Control of Fusarium wilt of radish by combining Pseudomonas putida strains that have different diseasesuppressive mechanisms. Phytopathology 93:626-632.

7. Dooley, S. R., and Beckstead, J. 2010. Characterizing the interaction between a fungal seed pathogen and a deleterious rhizobacterium for biological control of cheatgrass. Biol. Control 53:197-203.

8. Elad, Y. 2000. Biological control of foliar pathogens by means of Trichoderma harzianum and potential modes of action. Crop Prot. 19:709-714.

9. Elad, Y, and Freeman, S. 2002. Biological control of fungal plant pathogens. In: The Mycota XI: Agricultural Applications. Kempken, ed. Springer-Verlag, Berlin.

10. Elad, Y., and Stewart, A. 2007. Microbial control of Botrytis spp. Pages 223-241 in: Botrytis: Biology, Pathology and Control. Y. Elad, B. Williamson, P. Tudzynski, and N. Delen, eds. Kluwer Academic Publishers, Dordrecht, The Netherlands.

11. Elliott, M., Shamoun, S. F., Sumampong, G., James, D., Masri, S., and Varga, A. 2009. Evaluation of several commercial biocontrol products on European and North American populations of Phytophthora ramorum. Biocontrol Sci. Technol. 19:1007-1021.

12. Elmer, P. A. G, and Köhl, J. 1998. The survival and saprophytic competitive ability of the Botrytis spp. antagonist Ulocladium atrum in lily canopies. Eur. J. Plant Pathol. 104:435-447.

13. Freeman, S., Minz, D., Kolesnik, I., Barbul, O., Zveibil, A., Maymon, M.,
Nitzani, Y., Kirshner, B., Rav-David, D., Bilu, A., Dag, A., Shafir, S., and Elad, Y. 2004. Trichoderma biocontrol of Colletotrichum acutatum and Botrytis cinerea and survival in strawberry. Eur. J. Plant Pathol. 110:361370 .

14. Garrett, K. A., and Mundt, C. C. 2000. Host diversity can reduce potato late blight severity for focal and general patterns of primary inoculum. Phytopathology 90:1307-1312.

15. Gilardi, G., Manker, D. C., Garibaldi, A., and Gullino, M. L. 2008. Efficacy of the biocontrol agents Bacillus subtilis and Ampelomyces quisqualis applied in combination with fungicides against powdery mildew of zucchini. J. Plant Dis. Prot. 115:208-213.

16. Gubbins, S., and Gilligan, C. A. 1997. A test of heterogeneous mixing as a mechanism for ecological persistence in a disturbed environment. Proc. R. Soc. London B 264:623-623.

17. Guetsky, R., Shtienberg, D., Elad, Y., and Dinoor, A. 2001. Combining biocontrol agents to reduce the variability of biological control. Phytopathology 91:621-627.

18. Guetsky, R., Shtienberg, D., Elad, Y., Fischer, E., and Dinoor, A. 2002. Improving biological control by combining biocontrol agents each with several mechanisms of disease suppression. Phytopathology 92:976-985.

19. Harman, G. E., Howell, C. R., Viterbo, A., Chet, I., and Lorito, M. 2004. Trichoderma species-opportunistic, avirulent plant symbionts. Nat. Rev. Microbiol. 2:43-56.

20. Janousek, C. N., Lorber, J. D., and Gubler, W. D. 2009. Combination and rotation of bacterial antagonists to control powdery mildew on pumpkin. J. Plant Dis. Prot. 116:260-262.

21. Jeger, M. J., Jeffries, P., Elad, Y., and Xu, X. M. 2009. A generic theoretical model for biological control of foliar plant diseases. J. Theor. Biol. 256:201-214.

22. Johnson, K. B. 2010. Pathogen refuge: A key to understanding biological control. Annu. Rev. Phytopathol. 48:141-160.

23. Kapat, A., Zimand, G., and Elad, Y. 1998. Effect of two isolates of Trichoderma harzianum on the activity of hydrolytic enzymes produced by Botrytis cinerea. Physiol. Mol. Plant Pathol. 52:127-137.

24. Kessel, G. J. T., De Haas, BH, Van Der Werf, W, and Köhl, J. 2002. Competitive substrate colonisation by Botrytis cinerea and Ulocladium atrum in relation to biological control of B. cinerea in cyclamen Mycol. Res. 106:716-728.

25. Kessel, G. J. T., Kohl, J., Powell, J. A., Rabbinge, R., and van der Werf, W. 2005. Modeling spatial characteristics in the biological control of fungi at leaf scale: Competitive substrate colonization by Botrytis cinerea and the saprophytic antagonist Ulocladium atrum. Phytopathology 95:439-448.

26. Knudson, G. R., and Hudler, G. W. 1987. Use of a computer simulation model to evaluate a plant disease biocontrol agent. Ecol. Model. 35:45-62.

27. Lannou, C., Hubert, P., and Gimeno, C. 2005. Competition and interactions among stripe rust pathotypes in wheat-cultivar mixtures. Plant Pathol. 54:699-712.

28. Le Floch, G., Tambong, J., Vallance, J., Tirilly, Y., Levesque, A., and Rey, P. 2007. Rhizosphere persistence of three Pythium oligandrum strains in tomato soilless culture assessed by DNA macroarray and real-time PCR. FEMS Microbiol. Ecol. 61:317-326.

29. Le Floch, G., Vallance, J., Benhamou, N., and Rey, P. 2009. Combining the oomycete Pythium oligandrum with two other antagonistic fungi: Root relationships and tomato grey mold biocontrol. Biol. Control 50:288-298.

30. Mitchell, J. K., Jeger, M. J., and Taber, R. A. 1987. The effect of temperature on colonisation of Cercosporidium personatum leaf spot of peanuts by the hyperparasite Dicyma pulvinata. Agric. Ecosyst. Environ. 18:325-332.

31. Monier, J. M., and Lindow, S. E. 2004. Frequency, size, and localization of bacterial aggregates on bean leaf surfaces. Appl. Environ. Microbiol. 70:346-355.

32. Monier, J. M., and Lindow, S. E. 2005. Spatial organization of dualspecies bacterial aggregates on leaf surfaces. Appl. Environ. Microbiol. 71:5484-5493.

33. Mundt, C. C. 2002. Performance of wheat cultivars and cultivar mixtures in the presence of Cephalosporium stripe. Crop Prot. 21:93-99.

34. Newton, A.C. 1997. Cultivar mixtures in intensive agriculture. Pages 6580 in: The Gene-for-Gene Relationship in Plant-Parasite Interactions. I. R. Crute, E. B. Holub, and J. J. Burdon eds. CAB International, Wallingford, Oxon, UK.

35. Paulitz, T. C., and Belanger, R. R. 2001. Biological control in greenhouse systems. Annu. Rev. Phytopathol. 39:103-133.

36. Punja, Z. K., and Utkhede, R. S. 2003. Using fungi and yeasts to manage vegetable crop diseases. Trends Biotechnol. 21:400-407.

37. Robinson-Boyer, L., Jeger, M., Xu, X.-M., and Jeffries, P. 2009. Management of strawberry grey mould using mixtures of biocontrol agents with different mechanisms of action. Biocontrol Sci. Technol. 19:1051-1065. 
38. Seaman, A. 2003. Efficacy of OMRI-approved products for tomato foliar disease control. N. Y. State Integr. Pest Manage. Program Publ. 129:164167.

39. Szczech, M., and Dyki, B. 2007. Combination of microbial biocontrol agents to control Rhizoctonia damping-off and Fusarium wilt of tomato. Bull. OILB/SROP 30:415-418.

40. Szczech, M., and Shoda, M. 2004. Biocontrol of Rhizoctonia damping-off of tomato by Bacillus subtilis combined with Burkholderia cepacia. J. Phytopathol. 152:549-556.

41. Taylor, D. R., Jarosz, A. M., Lenski, R. E., and Fulbright, D. W. 1998. The acquisition of hypovirulence in host-pathogen systems with three trophic levels. Am. Nat. 154:343-355.

42. van den Bosch, F., and Gilligan, C. A. 2008. Models of fungicide resistance dynamics. Annu. Rev. Phytopathol. 46:123-147.

43. Whipps, J. M. 2001. Microbial interactions and biocontrol in the rhizosphere. J. Exp. Bot. 52:487-511.

44. Xu, X.-M., Jeffries, P., Pautasso, M., and Jeger, M. J. 2011. Combined use of biocontrol agents to manage plant diseases in theory and practice.
Phytopathology 101:1024-1031.

45. Xu, X.-M., Robinson, J. D, Jeger, M, and Jeffries, P. 2010. Using combinations of biocontrol agents to control Botrytis cinerea on strawberry leaves under fluctuating temperatures. Biocontrol Sci. Technol. 20:359-373.

46. Xu, X.-M., Salama, N., Jeffries, P., and Jeger, M. J. 2010. Numerical studies of biocontrol efficacies of foliar plant pathogens in relation to the characteristics of a biocontrol agent. Phytopathology 100:814-821.

47. Yedidia, I, Benhamou, N, Kapulnik, Y, and Chet, I. 2000. Induction and accumulation of PR proteins activity during early stages of root colonization by the mycoparasite Trichoderma harzianum strain T-203. Plant Physiol. Biochem. 38:863-873.

48. Yobo, K. S., Laing, M. D., and Hunter, C. H. 2010. Application of selected biological control agents in conjunction with tolclofos-methyl for the control of damping-off caused by Rhizoctonia solani. Afr. J. Biotechnol. 9:1789-1796.

49. Yu, H., and Sutton, J. C. 1999. Density dynamics of Gliocladium roseum in relation to biological control of Botrytis cinerea in red raspberry. Can. J. Plant Pathol. 21:23-32. 